\title{
IMPROVED PREDICTION FOR MEDIUM OPTIMIZATION USING FACTORIAL SCREENING FOR DOCOSAHEXAENOIC ACID PRODUCTION BY SCHIZOCHYTRIUM SP. SW1
}

\author{
${ }^{1}$ Vidyah Manikan, ${ }^{2}$ Mohd Sahaid Kalil, ${ }^{3}$ Mohd Hafez Mohd Isa and ${ }^{1}$ Aidil Abdul Hamid \\ ${ }^{1}$ Faculty of Science and Technology, Universiti Kebangsaan Malaysia, Malaysia \\ ${ }^{2}$ Department of Chemical and Process Engineering, Faculty of Engineering and Built Environment, \\ Universiti Kebangsaan Malaysia, Malaysia \\ ${ }^{3}$ Faculty of Science and Technology, Universiti Sains Islam Malaysia, Malaysia
}

Received 2013-08-14; Revised 2013-12-19; Accepted 2014-01-15

\begin{abstract}
Docosa Hexaenoic Acid (DHA) is an essential fatty acid that plays major role in human health. It is typically extracted from fatty fish and this puts pressure on global fish stock. DHA obtained from fish is also prone to contamination. Schizochytrium is a potential alternative source, having the ability of accumulating considerable amounts of DHA. Modification of medium components and culture conditions are the preferred techniques to enhance DHA production by this marine microalga. In this study, two-level factorial design was employed to investigate the significance of sea salt, glucose, yeast extract, Monosodium Glutamate (MSG) and peptone in affecting the biomass, lipid and DHA accumulation processes of a locally isolated strain of Schizochytrium. In contrast to the conventional ANOVA method commonly employed in various screening studies, half normal probability plots were used to identify the significant factors, whereas interaction plots were used to explain the pattern of interaction between significant combinations. Design Expert software was used to construct a set of experiments where each medium component mentioned above was varied over two levels. Cultivation was carried out in $250 \mathrm{~mL}$ flasks containing $50 \mathrm{~mL}$ of medium, incubated at $30^{\circ} \mathrm{C}$ with $200 \mathrm{rpm}$ agitation for $96 \mathrm{~h}$. This experiment revealed that sea salt and interaction of glucose-MSG have significant effect on biomass production whereas sea salt and interaction of sea salt-glucose affected lipid accumulation. Meanwhile, interactions of glucose-yeast extract and glucose-MSG have significant effect on DHA accumulation. Detailed analysis of interaction plots enabled deeper understanding of the behavior of the system in response to the significant variables, which improves the precision in determination of variable domains for effective medium optimization.
\end{abstract}

Keywords: Schizochytrium, Docosahexaenoic Acid, Screening of Medium Components, Factorial Design

\section{INTRODUCTION}

Long-chain omega- 3 polyunsaturated fatty acids $(n 3$ LC-PUFAs), Especially Eicosapentaenoic Acid (EPA, C20:5n3) and Docosahexaenoic Acid (DHA, C22:6n3) are two important fatty acids in human metabolism. DHA occurs naturally in breast milk and is essential for normal infant brain and eye development (Fedorova et al., 2011). It has also been proven to be useful in prevention and treatment of human diseases such as heart and inflammatory disorders (Perez-Garcia et al., 2011). Due to its significant role in enhancement of human health, DHA is widely used as a nutraceutical component in the food and feed market (Zeng et al., 2011). The present largest and richest commercial source of DHA is cold water fatty fish, such as salmon, sardine, anchovy, tuna and cod which can produce up to $30 \%$ of DHA and EPA (Vazhappilly and Chen, 1998). In fish oils, both composition and quantity of PUFA depend on the species and other external factors (Shene et al., 2010). In addition, fish oil usually contains considerable amounts of highly saturated fatty acids and its PUFA profile is Corresponding Author: Aidil Abdul Hamid, Faculty of Science and Technology, Universiti Kebangsaan Malaysia, Malaysia 
complex. This causes complications in purification processes which in turn, increases the production cost (Wu et al., 2005). Moreover, there are emerging concerns with sustainability of marine resources and with the levels of environmental contaminants (PCBs, dioxins and mercury) in fish. Thus, major efforts have been made to identify or create alternative sources as the current sole main source of DHA may not provide a steady supply for the increasing market demands (Chi et al., 2009; Hauvermale et al., 2006). Since fish obtain n-3 fatty acids from zooplankton which consumes algae, studies now are being diverted to production of DHA directly from its primary source, microalgae (Vazhappilly and Chen, 1998).

Several species of microalgae can be induced to overproduce specific fatty acids through relative simple manipulations of the physical and chemical properties of their culture medium. By manipulating fatty acid content, microalgae represent a significant source of unusual and valuable lipids and fatty acids for numerous industrial applications (Behrens and Kyle, 1996). Thus, microalgae that contain large quantities of high-quality DHA are considered a potential alternative to fish oil, especially under heterotrophic conditions that reduce the costs of production (Barclay et al., 1994; Chi et al., 2007). It has been shown that microalgae-based heterotrophic production systems can exhibit omega-3 fatty acid productivities that are two to three orders of magnitude greater than those of outdoor autotrophic pond systems. Additionally, long-chain n-3 fatty acid productivities reported for the microalgae fermentation systems are one to two orders of magnitude greater than productivities reported for fungal or bacterial systems (Barclay et al., 1994).

Marine fungoid protists (Thraustochytrids) such as Schizochytrium and marine microalgae such as Crypthecodinium cohnii have been shown to be excellent DHA producers. Members of the genus Schizochytrium, as well as other strains of Thraustochytrids can produce large amounts of oil, which is up to $55 \%$ of the cell weight in which DHA can comprise as much as $35 \%$ of the total fatty acids. Schizochytrium sp. is grown on a commercial scale via fermentation for both biomass (for animal feed) and for its oil (Barclay et al., 2005). DHA from Schizochytrium has also been proven to be safe for human consumption, being free from the common algal toxins such as domoic acid and prymnesin produced by some members of its kingdom, Chromista (Ratledge, 2003).

Lately, statistical Design of Experiments (DoE) has gained better recognition in screening and optimization experiments. It is useful to identify the important factors and interactions between two or more variables in relatively few experiments as compared to the conventional onefactor-at-time technique (Tumpang et al., 2011). The twolevel factorial design can be considered to be a multivariable sequential search technique in which the effects of two or more factors are studied simultaneously and the responses are analyzed statistically to arrive at a decision (Duta et al., 2006; Anbu et al., 2006). However, this technique is given less attention compared to optimization processes. Detailed analysis of factorial screening results can be very helpful in discovering the right combination of factors that allows the microbial system to achieve optimal production. Elimination of insignificant factors prior to optimization procedure saves the cost of fermentation. When this is done, statistical model(s) obtained in optimization step provides better estimations which lead to more robust solutions. Even though response surface analyses also allow identification of insignificant factors, inclusion of these factors in the experimental runs often leads to imprecise estimations and relatively large residual error. Consequently, false optimal combinations are suggested at the end of the process. Therefore, it is crucial to perform a factorial screening experiment prior to optimization step.

Common approaches in factorial screening often disregard the analysis of interaction plots, while giving more attention to ANOVA to determine the significance of variables. Consequently, the behavior of the system in response to the interactions between significant variables is not well understood. Thorough analysis of the interaction plots enables better understanding of the system which in turn leads to precise determination of the ranges of variables for further processes, such as optimization. In this study, five medium components were screened for their level of significance in affecting biomass, lipid and DHA production by a locally isolated strain of Schizochytrium (GenBank KF500513). Half normal probability plots were used to identify the significant factor(s) and interaction plots were used to visualize the pattern of interaction between the factors and the combined effect on responses. Models and significance levels of variables were validated using Analysis of Variance (ANOVA).

\section{MATERIALS AND METHODS}

\subsection{Organism and Culture Conditions}

Culture of SW1 was obtained from a microbial physiology lab in the School of Biosciences and Biotechnology, Universiti Kebangsaan Malaysia. 
It was maintained at room temperature on Seawater Nutrient Agar (SNA) slant which contained $28 \mathrm{~g} \mathrm{~L}^{-1}$ nutrient agar and $17.5 \mathrm{~g} \mathrm{~L}^{-1}$ sea salt. Two strips of SNA containing approximately 5 colonies ( $48 \mathrm{~h}$ old) each were used to inoculate $50 \mathrm{~mL}$ seeding broth (in $250 \mathrm{~mL}$ flasks) containing $60 \mathrm{~g} \mathrm{~L} \mathrm{~L}^{-1}$ glucose (sterilized and added separately), $2 \mathrm{~g} \mathrm{~L}^{-1}$ yeast extract, $8 \mathrm{~g} \mathrm{~L}^{-1}$ Monosodium Glutamate (MSG) and $6 \mathrm{~g} \mathrm{~L}^{-1}$ sea salt. The seed culture was then incubated for $48 \mathrm{~h}$ with $200 \mathrm{rpm}$ agitation at $30^{\circ} \mathrm{C}$.

A $10 \% \mathrm{v} / \mathrm{v}$ inoculum was inoculated into $50 \mathrm{~mL}$ production medium containing sea salt, glucose, yeast extract, MSG and peptone according to the levels set in the experimental design. The cultures were incubated for $96 \mathrm{~h}$ at $30^{\circ} \mathrm{C}$ with $200 \mathrm{rpm}$ agitation.

\subsection{Experimental Design}

Two-level factorial design with resolution $\mathrm{V}$ was used to screen for the significant factors among sea salt, glucose, yeast extract, MSG and peptone concentrations. Table 1 shows the details of the design. Confidence level was set at $5 \%$ and therefore the variables which scored a Probability $(\mathrm{P})$ value less than 0.05 were considered as influential factors affecting the response.
Experiment was designed using Design expert software (Stat-Ease Inc., Version 6.0.4). The design contained a total of 16 runs. Results presented in Table 2 are the average values for duplicative runs.

\subsection{Determination of Dry Cell Weight}

Cells were harvested by filtration using Whatman no. 1 filter papers followed by rinsing twice with $50 \mathrm{ml}$ sterile distilled water. Samples were oven-dried at $95^{\circ} \mathrm{C}$ to constant weight. Biomass was expressed as oven-dried weight in gram per liter of growth medium.

\subsection{Lipid Extraction and Fatty Acid Analysis}

Lipid extraction was done using Folch method (Folch et al., 1957). The extract was vaporized at room temperature and dried in vacuum desiccator until the weight was constant. Fatty acid compositions of the samples were determined as Fatty Acid Methyl Ester (FAME) by gas chromatography (HP 5890) equipped with a capillary column (BPX 70, $30 \mathrm{~m}, 0.32$ $\mu \mathrm{m})$ at $200^{\circ} \mathrm{C}$ using helium as a carrier gas with flow rate of $40 \mathrm{~cm}^{3} \mathrm{~min}^{-1}$. FAME was prepared by dissolving $0.05 \mathrm{~g}$ of the sample in $0.95 \mathrm{~mL}$ hexane and the mixture was added to $0.05 \mathrm{~mL}$ of $1 \mathrm{M}$ sodium methoxide.

Table 1. Range of factors for two level factorial analysis

\begin{tabular}{llr}
\hline Factor & Low level $(-1)$ & High level $(+1)$ \\
A: Sea salt $(\mathrm{g} / \mathrm{L})$ & 0.00 & 35.00 \\
B: Glucose $(\mathrm{g} / \mathrm{L})$ & 5.00 & 60.00 \\
C: Yeast extract $(\mathrm{g} / \mathrm{L})$ & 1.00 & 4.00 \\
D: MSG $(\mathrm{g} / \mathrm{L})$ & 0.00 & 16.00 \\
E: Peptone $(\mathrm{g} / \mathrm{L})$ & 0.00 & 2.00 \\
\hline
\end{tabular}

Table 2. Two level factorial design and experimental response according to the run order

\begin{tabular}{|c|c|c|c|c|c|c|c|c|}
\hline Run & $\begin{array}{l}\text { A: Sea } \\
\text { salt }\end{array}$ & $\begin{array}{l}\text { B: } \\
\text { Glucose }\end{array}$ & $\begin{array}{l}\text { C: Yeast } \\
\text { extract }\end{array}$ & $\begin{array}{l}\text { D: } \\
\text { MSG }\end{array}$ & $\begin{array}{l}\text { E: } \\
\text { Peptone }\end{array}$ & $\begin{array}{l}\text { Dry biomass } \\
(\mathrm{g} / \mathrm{L})\end{array}$ & $\begin{array}{l}\text { Lipid } \\
\text { (\% g/g biomass) }\end{array}$ & $\begin{array}{l}\text { DHA } \\
(\% \text { g/g lipid) }\end{array}$ \\
\hline 1 & 1 & -1 & 1 & 1 & -1 & 17.74 & 3.38 & 13.95 \\
\hline 2 & 1 & -1 & 1 & -1 & 1 & 7.00 & 4.29 & 37.93 \\
\hline 3 & 1 & 1 & -1 & -1 & 1 & 15.48 & 23.26 & 6.60 \\
\hline 4 & -1 & -1 & 1 & -1 & -1 & 0.58 & 31.03 & 48.22 \\
\hline 5 & -1 & 1 & 1 & -1 & 1 & 2.56 & 6.25 & 7.60 \\
\hline 6 & 1 & -1 & -1 & 1 & 1 & 28.64 & 5.10 & 5.96 \\
\hline 7 & 1 & -1 & -1 & -1 & -1 & 11.78 & 2.72 & 28.74 \\
\hline 8 & 1 & 1 & -1 & 1 & -1 & 3.30 & 7.88 & 29.96 \\
\hline 9 & 1 & 1 & 1 & -1 & -1 & 20.12 & 17.79 & 4.47 \\
\hline 10 & 1 & 1 & 1 & 1 & 1 & 29.20 & 13.49 & 16.44 \\
\hline 11 & -1 & 1 & -1 & 1 & 1 & 2.58 & 10.85 & 33.44 \\
\hline 12 & -1 & -1 & 1 & 1 & 1 & 2.58 & 21.71 & 28.19 \\
\hline 13 & -1 & 1 & -1 & -1 & -1 & 2.44 & 37.70 & 11.05 \\
\hline 14 & -1 & 1 & 1 & 1 & -1 & 1.60 & 16.25 & 7.99 \\
\hline 15 & -1 & -1 & -1 & 1 & -1 & 2.46 & 45.53 & 2.62 \\
\hline 16 & -1 & -1 & -1 & -1 & 1 & 0.24 & 73.91 & 7.01 \\
\hline
\end{tabular}




\section{RESULTS}

Screening step is crucial in eliminating the medium components that do not play critical role in promoting the accumulation of desired product. It also helps to identify components that have adverse effects on the production levels. This ensures that the growth condition provided is favorable for the microbe to produce and accumulate the product of interest in good amounts. In our preliminary study, SW1 achieved intensive growth when cultivated in a medium composed of glucose, yeast extract, MSG and sea salt, while percentage of DHA accumulation was highest in another medium which was composed of glucose, yeast extract, peptone and sea salt. Therefore, attempts were made to identify significant medium components and its ranges that would promote high biomass, lipid and DHA accumulation by Schizochytrium SW1 using factorial design.

In this study, every factor is varied over two levels, high and low, indicated by +1 and -1 respectively. The analysis gives an overall estimation on the effects of each variable as an independent factor and in combination with other factors. Design resolution was set at level V, in which the number of runs is half of that found in full factorial design. For all three responses, base $10 \mathrm{log}$ power transformation was applied to better predict the growth related data. This has also increased the normality of the data which in turn increases the applicability and usefulness of statistical techniques that are primarily based on the normality assumption. The statistical assumption of normal distribution of residuals and independency of residual with constant variance were validated separately for each response using the normal plot of residuals and "residual versus predicted" plots.

\subsection{Effect of Medium Components on Biomass}

The biomass production by SW1 was varied over a wide range, which is from 0.24 to $29.2 \mathrm{~g} \mathrm{~L}^{-1}$ culture medium. Table 3 shows the complete matrix for this response. A, B, C, D, E and $\mathrm{Y}_{1}$ are sea salt, glucose, yeast extract, MSG, peptone and biomass ( $\mathrm{g} / \mathrm{L})$ respectively.

From this table, it could be seen that sea salt (A) has a significant effect on biomass where its effect (studentized) is far greater than that of the other factors. Thus, sea salt is affecting the biomass strongly on a positive scale where its presence at high levels promotes the cell growth. The next highest effect value is scored by interaction effects of glucose and MSG (BD) which is greater on an absolute scale than its parent effect (B and
D). This shows that the combination of glucose and MSG produces a big negative impact on the biomass. The other effects range from 0.03 to 0.26 on an absolute value scale. These values are usually assumed to be due to normal variations. However, a normal plot (or half normal plot) is needed to verify this assumption. Figure 1 shows the half normal plot for the effects on the biomass.

In Fig. 1, effects (factors) that are at considerable distance from the rest of the points are considered to be significant statistically. This is in agreement with the studentized effect values as discussed above, where effects of A and BD scored higher values than the rest of the factors which represent normal scatter. This pattern is typical, where majority of the points fall in a line emanating from the origin (Anderson and Whitcomb, 2007). Interaction between glucose and MSG can be illustrated using an interaction plot as shown in Fig. 2.

Bar brackets at the ends of the lines indicate Least Significant Difference (LSD). Overlapping of LSD bars shows the significance of the factor. In Fig. 2, these bars do not overlap for both high and low levels (D+ and D-), again showing that the levels of glucose influence the biomass significantly. Parallel lines indicate that there is no interaction and nonparallel lines as seen in Fig. 2 are characteristics of a powerful two-factor interaction (Anderson and Whitcomb, 2007). The effect of glucose on the biomass depends on the level of MSG, represented by the two lines on the plot. When MSG is at low level, increasing glucose concentrations has positive effects on the biomass, whereas when MSG is at high level, increasing glucose concentrations is unfavorable for this response. Thus, is can be concluded that providing high or low amounts of both glucose and MSG is not desirable for high biomass production of Schizochytrium SW1.

\subsection{Effect of Medium Components on Lipid}

The lowest amount of lipid accumulated was $2.72 \%$ whereas the highest was $73.91 \% \mathrm{~g} / \mathrm{g}$ biomass. Table 4 shows the matrix for the second response, $\mathrm{Y}_{2}$ which is lipid/biomass in percentage.

Based on the studentized effects shown in Table 4, factor A (sea salt) and $\mathrm{AB}$ (interaction of sea salt and glucose) are significant factors, affecting the amount of lipid accumulated per unit biomass. The negative value of effects for A implies that this component affects the percentage of lipid accumulation adversely in contrast to its effect on biomass discussed above. Thus, concentration of sea salt to be incorporated into the medium must be carefully chosen to obtain optimal amounts of both biomass and lipid. 
Vidyah Manikan et al. / American Journal of Applied Sciences 11 (3): 462-474, 2014

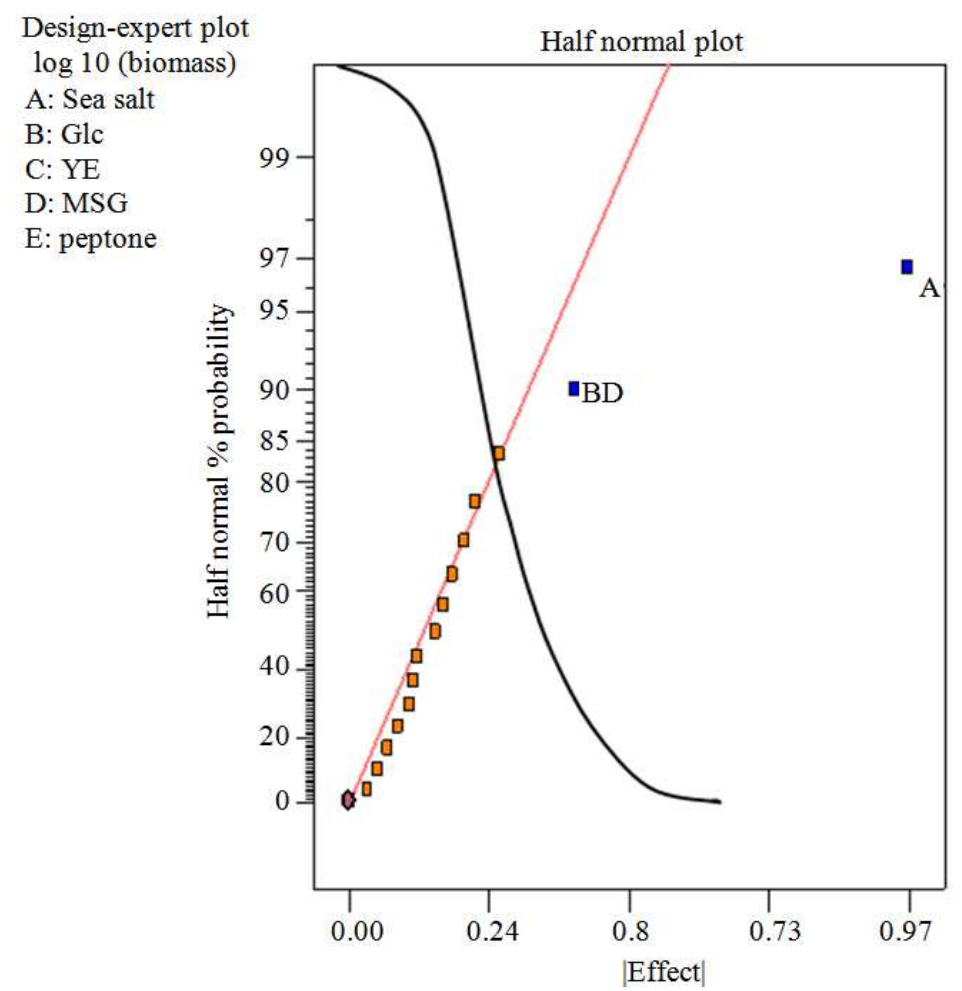

Fig. 1. Half normal dot-plot of effects for biomass

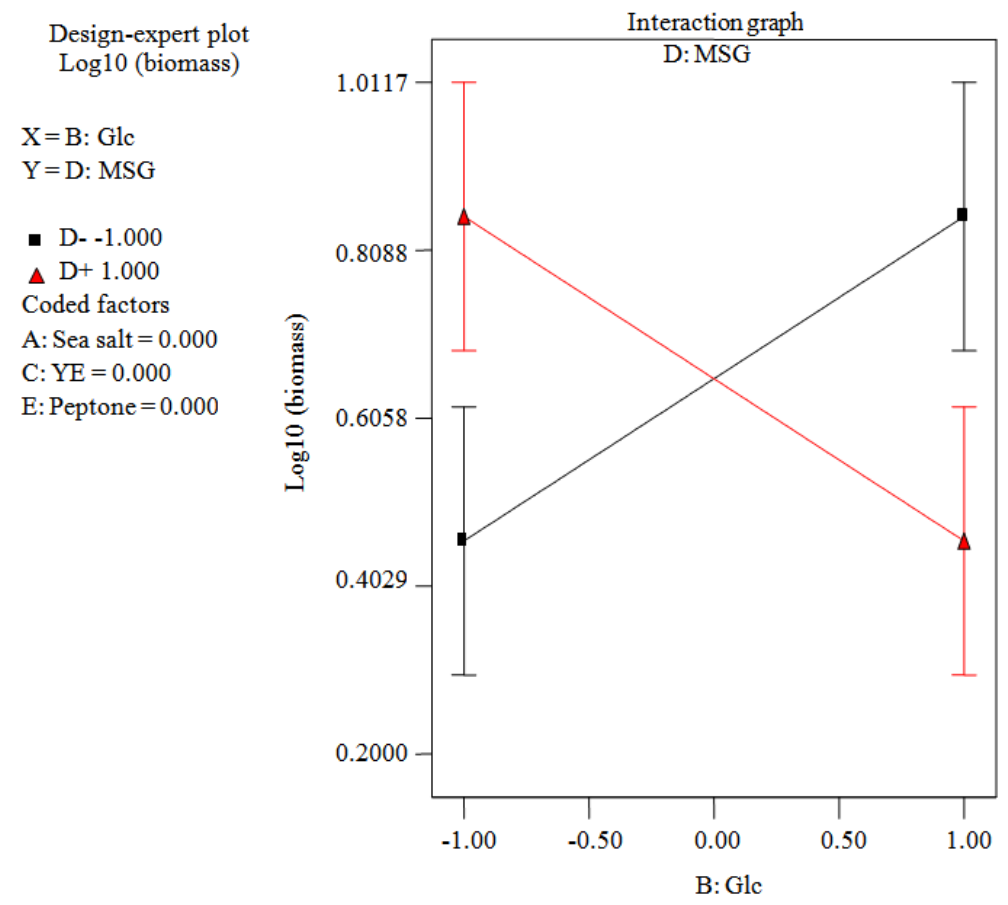

Fig. 2. Interaction effect of glucose versus MSG on biomass 
Table 3. Complete matrix for biomass

\begin{tabular}{|c|c|c|c|c|c|c|c|c|c|c|c|c|c|c|c|c|}
\hline \multirow[b]{2}{*}{ Std } & \multicolumn{8}{|c|}{ Main effects } & \multicolumn{8}{|c|}{ Interaction effects } \\
\hline & A & $\mathrm{B}$ & $\mathrm{C}$ & $\mathrm{D}$ & $\mathrm{E}$ & $\mathrm{AB}$ & $\mathrm{AC}$ & $\mathrm{AD}$ & $\mathrm{AE}$ & $\mathrm{BC}$ & $\mathrm{BD}$ & $\mathrm{BE}$ & $\mathrm{CD}$ & $\mathrm{CE}$ & $\mathrm{DE}$ & $\mathrm{Y}_{1}$ \\
\hline 1 & - & - & - & - & + & + & + & + & - & + & + & - & + & - & - & 0.24 \\
\hline 2 & + & - & - & - & - & - & - & - & - & + & + & + & + & + & + & 11.78 \\
\hline 3 & - & + & - & - & - & - & + & + & + & - & - & - & + & + & + & 2.44 \\
\hline 4 & + & + & - & - & + & + & - & - & + & - & - & + & + & - & - & 15.48 \\
\hline 5 & - & - & + & - & - & + & - & + & + & - & + & + & - & - & + & 0.58 \\
\hline 6 & + & - & + & - & + & - & + & - & + & - & + & - & - & + & - & 7.00 \\
\hline 7 & - & + & + & - & + & - & - & + & - & + & - & + & - & + & - & 2.56 \\
\hline 8 & + & + & + & - & - & + & + & - & - & + & - & - & - & - & + & 20.12 \\
\hline 9 & - & - & - & + & - & + & + & - & + & + & - & + & - & + & - & 2.46 \\
\hline 10 & + & - & - & + & + & - & - & + & + & + & - & - & - & - & + & 28.64 \\
\hline 11 & - & + & - & + & + & - & + & - & - & - & + & + & - & - & + & 2.58 \\
\hline 12 & + & + & - & + & - & + & - & + & - & - & + & - & - & + & - & 3.30 \\
\hline 13 & - & - & + & + & + & + & - & - & - & - & - & - & + & + & + & 2.58 \\
\hline 14 & + & - & + & + & - & - & + & + & - & - & - & + & + & - & - & 17.74 \\
\hline 15 & - & + & + & + & - & - & - & - & + & + & + & - & + & - & - & 1.60 \\
\hline 16 & + & + & + & + & + & + & + & + & + & + & + & + & + & + & + & 29.20 \\
\hline Effect & 0.97 & 0.17 & 0.11 & 0.22 & 0.09 & -0.2 & 0.05 & -0.15 & 0.12 & 0.11 & -0.39 & 0.18 & 0.03 & 0.07 & 0.26 & 9.27 \\
\hline
\end{tabular}

Table 4. Complete matrix for lipid

\begin{tabular}{|c|c|c|c|c|c|c|c|c|c|c|c|c|c|c|c|c|}
\hline \multirow[b]{2}{*}{ Std } & \multicolumn{8}{|c|}{ Main effects } & \multicolumn{8}{|c|}{ Interaction effects } \\
\hline & A & B & C & D & E & $\mathrm{AB}$ & $\mathrm{AC}$ & $\mathrm{AD}$ & $\mathrm{AE}$ & $\mathrm{BC}$ & $\mathrm{BD}$ & $\mathrm{BE}$ & CD & $\mathrm{CE}$ & $\mathrm{DE}$ & $\mathrm{Y}_{2}$ \\
\hline 1 & - & - & - & - & + & + & + & + & - & + & + & - & + & - & - & 73.91 \\
\hline 2 & + & - & - & - & - & - & - & - & - & + & + & + & + & + & + & 2.72 \\
\hline 3 & - & + & - & - & - & - & + & + & + & - & - & - & + & + & + & 37.70 \\
\hline 4 & + & + & - & - & + & + & - & - & + & - & - & + & + & - & - & 23.26 \\
\hline 5 & - & - & + & - & - & + & - & + & + & - & + & + & - & - & + & 31.03 \\
\hline 6 & + & - & + & - & + & - & + & - & + & - & + & - & - & + & - & 4.29 \\
\hline 7 & - & + & + & - & + & - & - & + & - & + & - & + & - & + & - & 6.25 \\
\hline 8 & + & + & + & - & - & + & + & - & - & + & - & - & - & - & + & 17.79 \\
\hline 9 & - & - & - & + & - & + & + & - & + & + & - & + & - & + & - & 45.53 \\
\hline 10 & + & - & - & + & + & - & - & + & + & + & - & - & - & - & + & 5.10 \\
\hline 11 & - & + & - & + & + & - & + & - & - & - & + & + & - & - & + & 10.85 \\
\hline 12 & + & + & - & + & - & + & - & + & - & - & + & - & - & + & - & 7.88 \\
\hline 13 & - & - & + & + & + & + & - & - & - & - & - & - & + & + & + & 21.71 \\
\hline 14 & + & - & + & + & - & - & + & + & - & - & - & + & + & - & - & 3.38 \\
\hline 15 & - & + & + & + & - & - & - & - & + & + & + & - & + & - & - & 16.25 \\
\hline 16 & + & + & & & + & + & + & + & + & + & & + & & & + & 13.49 \\
\hline Effect & -0.5 & 0.08 & -0.15 & -0.11 & -0.02 & 0.51 & 0.18 & 0 & 0.2 & 0.02 & -0.07 & -0.13 & 0.12 & -0.12 & 0 & 20.07 \\
\hline
\end{tabular}

Interactions of $\mathrm{AB}$ on the other hand exert positive effect on lipid accumulation. This interaction which involves sea salt might be helpful in determination of optimal level of this component which is of critical importance. Figure 3 is the plot of half normal probability for this response and Fig. 4 is the interaction plot illustrating the combined effect of sea salt and glucose concentrations on the percentage of lipid accumulated.

The LSD bars of glucose at +1 level are overlapping in this plot which signifies that effect of sea salt is not significant when glucose is at high level. However, at low level (B-), LSD bars of glucose do not overlap, indicating that the effect of sea salt is significant. Here, the line angles steeply downward, showing a strong negative effect due to the increased concentration of sea salt. This is clearly shown by the response where percentage of lipid is only 3.87 by average (standard order 2, 6, 10 and 14) when sea salt is at high level and glucose is at low level. When glucose is set at high level, the lipid content remains unchanged regardless of the levels of sea salt.

\subsection{Effect of Medium Components on DHA}

Highest amount of DHA (\% g/g lipid) was produced in run number 4 , where it accounts for $48.22 \%$ of the total lipid, whereas lowest amount was produced in run number $15(2.62 \%)$. The complete matrix for this response $\left(\mathrm{Y}_{3}\right)$ is shown in Table 5 . 
Vidyah Manikan et al. / American Journal of Applied Sciences 11 (3): 462-474, 2014

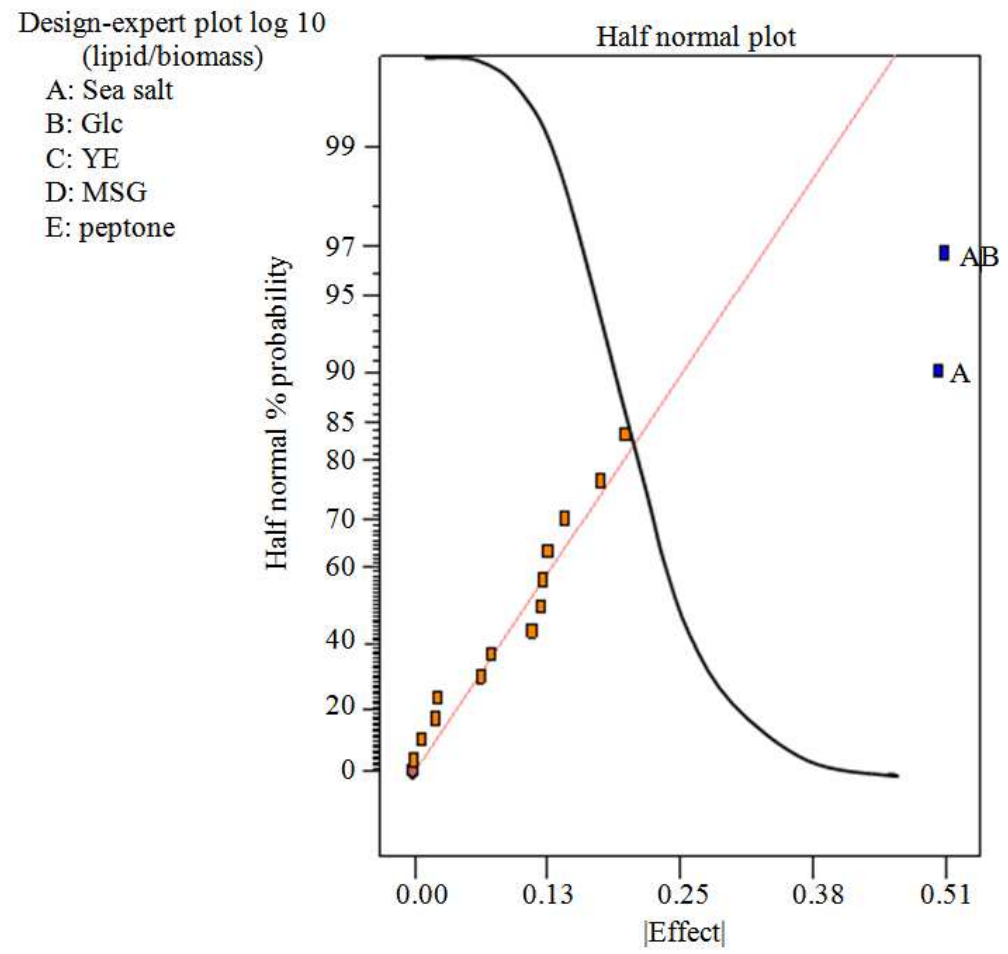

Fig. 3. Half normal dot-plot of effects for lipid

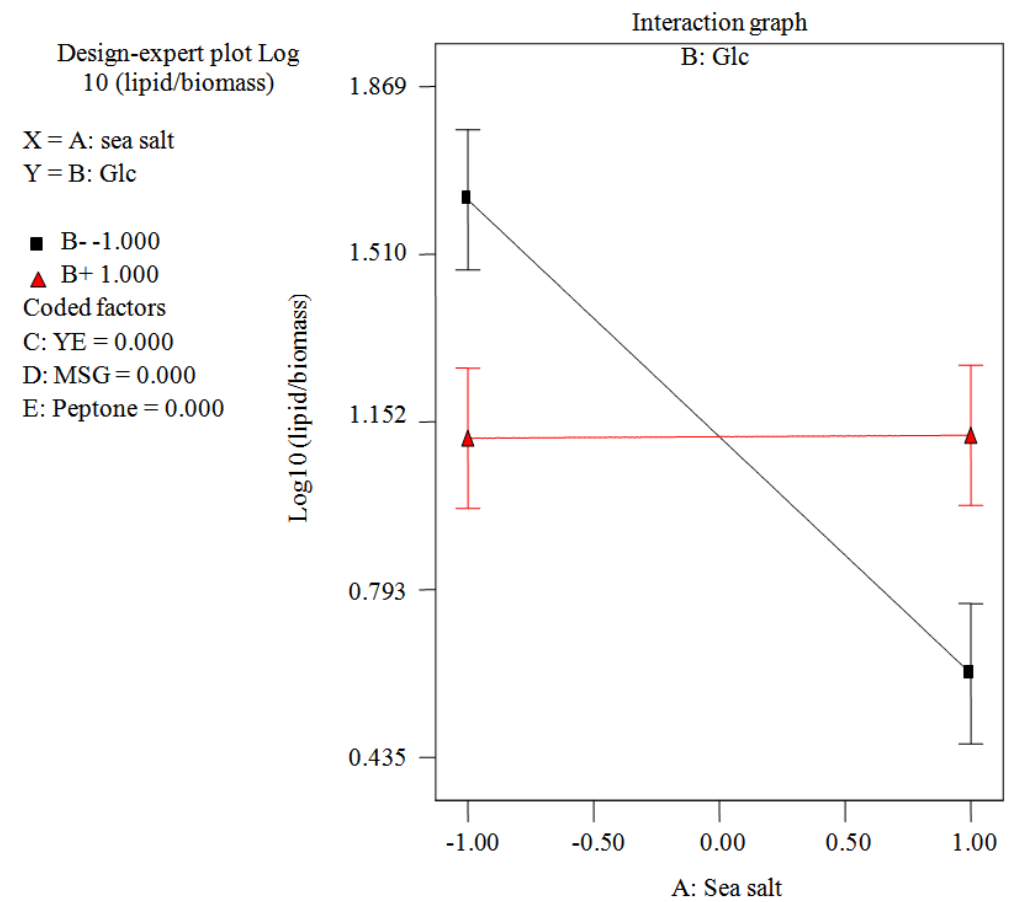

Fig. 4. Interaction effect of sea salt versus glucose on lipid 
Table 5. Complete matrix for DHA

\begin{tabular}{|c|c|c|c|c|c|c|c|c|c|c|c|c|c|c|c|c|}
\hline \multirow[b]{2}{*}{ Std } & \multicolumn{8}{|c|}{ Main effects } & \multicolumn{8}{|c|}{ Interaction effects } \\
\hline & A & $\mathrm{B}$ & $\mathrm{C}$ & $\mathrm{D}$ & $\mathrm{E}$ & $\mathrm{AB}$ & $\mathrm{AC}$ & $\mathrm{AD}$ & $\mathrm{AE}$ & $\mathrm{BC}$ & $\mathrm{BD}$ & $\mathrm{BE}$ & $\mathrm{CD}$ & $\mathrm{CE}$ & DE & Y3 \\
\hline 1 & - & - & - & - & + & + & + & + & - & + & + & - & + & - & - & 7.01 \\
\hline 2 & + & - & - & - & - & - & - & - & - & + & + & + & + & + & + & 28.74 \\
\hline 3 & - & + & - & - & - & - & + & + & + & - & - & - & + & + & + & 11.05 \\
\hline 4 & + & + & - & - & + & + & - & - & + & - & - & + & + & - & - & 6.60 \\
\hline 5 & - & - & + & - & - & + & - & + & + & - & + & + & - & - & + & 48.22 \\
\hline 6 & + & - & + & - & + & - & + & - & + & - & + & - & - & + & - & 37.93 \\
\hline 7 & - & + & + & - & + & - & - & + & - & + & - & + & - & + & - & 7.60 \\
\hline 8 & + & + & + & - & - & + & + & - & - & + & - & - & - & - & + & 4.47 \\
\hline 9 & - & - & - & + & - & + & + & - & + & + & - & + & - & + & - & 2.62 \\
\hline 10 & + & - & - & + & + & - & - & + & + & + & - & - & - & - & + & 5.96 \\
\hline 11 & - & + & - & + & + & - & + & - & - & - & + & + & - & - & + & 33.44 \\
\hline 12 & + & + & - & + & - & + & - & + & - & - & + & - & - & + & - & 29.96 \\
\hline 13 & - & - & + & + & + & + & - & - & - & - & - & - & + & + & + & 28.19 \\
\hline 14 & + & - & + & + & - & - & + & + & - & - & - & + & + & - & - & 13.95 \\
\hline 15 & - & + & + & + & - & - & - & - & + & + & + & - & + & - & - & 7.99 \\
\hline 16 & + & + & + & + & + & + & + & + & + & + & + & + & + & + & + & 16.44 \\
\hline Effect & 0.05 & -0.11 & 0.14 & -0.01 & 0.04 & -0.09 & -0.13 & 0.03 & -0.12 & -0.45 & 0.44 & 0.05 & -0.01 & 0.15 & 0.22 & 18.14 \\
\hline
\end{tabular}

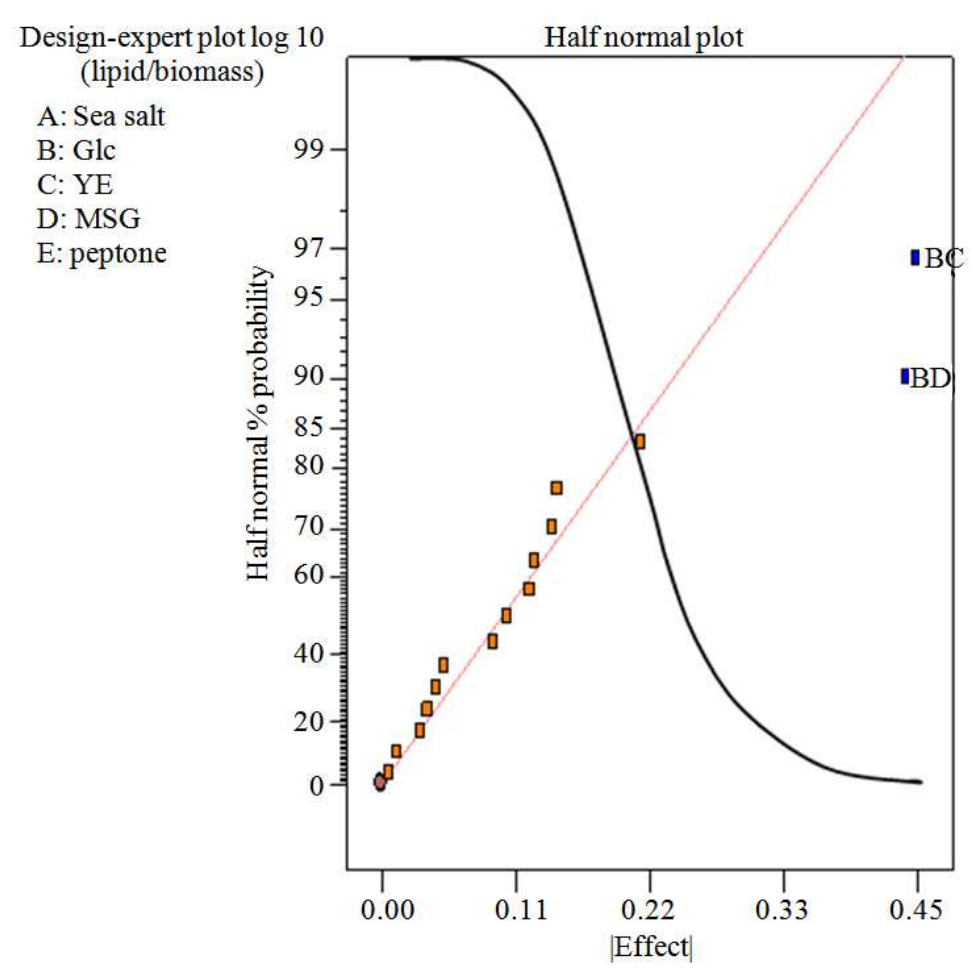

Fig. 5. Half normal dot-plot of effects for DHA

In contrast to the response of biomass and lipid, percentage of DHA is not affected significantly by any major factors. However, interactions of BC (glucose and yeast extract) and BD (glucose and MSG) have significant negative effect on the proportion of DHA in the accumulated lipid. Figure $\mathbf{5}$ shows the normal probability plot for DHA while Fig. 6 and 7 show the interaction graphs for the effects on $\mathrm{BC}$ and $\mathrm{BD}$ respectively. 
Vidyah Manikan et al. / American Journal of Applied Sciences 11 (3): 462-474, 2014

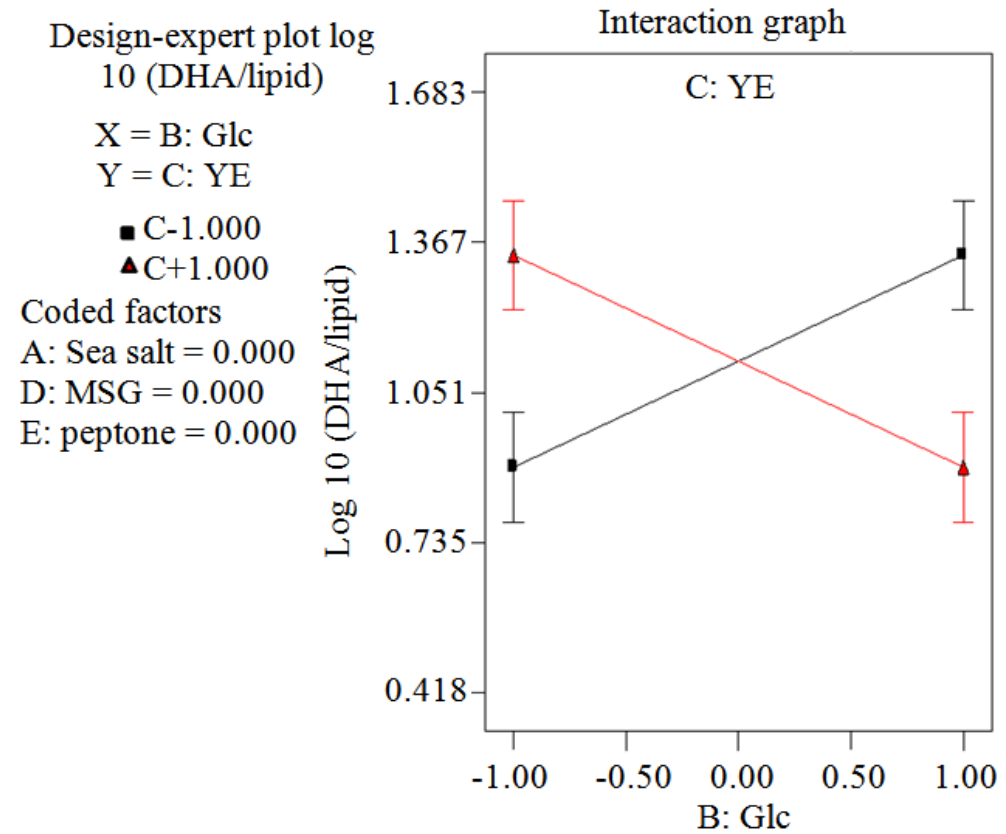

Fig. 6. Interaction effect of glucose versus yeast extract on DHA

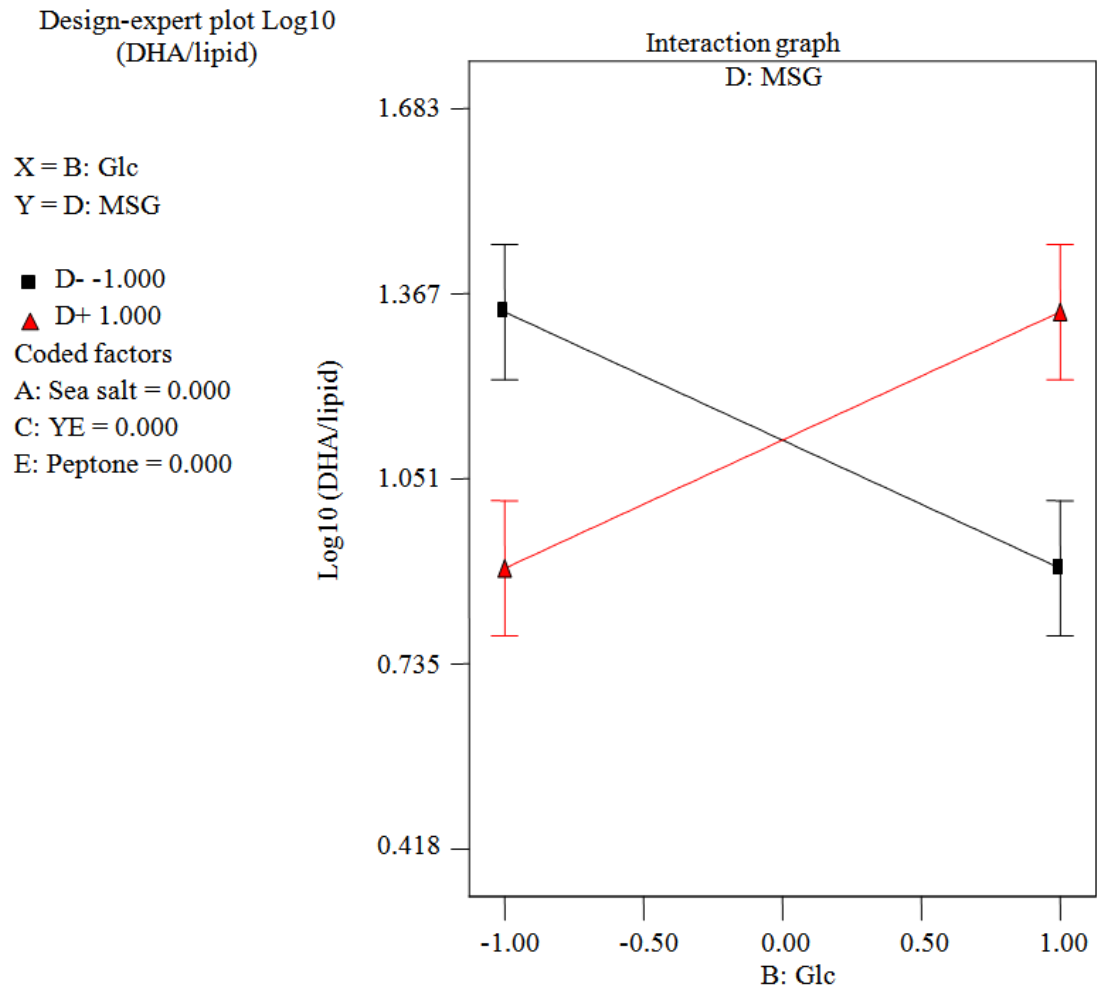

Fig. 7. Interaction effect of glucose and MSG on DHA 
Vidyah Manikan et al. / American Journal of Applied Sciences 11 (3): 462-474, 2014

Table 6. Combined ANOVA results for biomass, lipid and DHA

\begin{tabular}{|c|c|c|c|c|c|}
\hline Source & Sum of squares & $\mathrm{df}$ & Mean square & F value & $\mathrm{P}>\mathrm{F}$ \\
\hline \multicolumn{6}{|l|}{ Biomass } \\
\hline Model & 4.36 & 2 & 2.18 & 24.24 & $<0.0001$ \\
\hline A & 3.75 & 1 & 3.75 & 41.63 & $<0.0001$ \\
\hline $\mathrm{BD}$ & 0.62 & 1 & 0.62 & 6.84 & 0.0214 \\
\hline Residual & 1.17 & 13 & 0.09 & & \\
\hline Cor total & 5.53 & 15 & & & \\
\hline \multicolumn{6}{|l|}{ Lipid } \\
\hline Model & 2.05 & 2 & 1.030 & 20.01 & 0.0001 \\
\hline A & 1.01 & 1 & 1.010 & 19.77 & 0.0007 \\
\hline $\mathrm{AB}$ & 1.04 & 1 & 1.040 & 20.26 & 0.0006 \\
\hline Residual & 0.67 & 13 & 0.051 & & \\
\hline Cor total & 2.72 & 15 & & & \\
\hline \multicolumn{6}{|l|}{ DHA } \\
\hline Model & 1.57 & 2 & 0.780 & 17.12 & 0.0002 \\
\hline $\mathrm{BC}$ & 0.80 & 1 & 0.800 & 17.46 & 0.0011 \\
\hline $\mathrm{BD}$ & 0.77 & 1 & 0.770 & 16.78 & 0.0013 \\
\hline Residual & 0.59 & 13 & 0.046 & & \\
\hline Cor total & 2.16 & 15 & & & \\
\hline
\end{tabular}

The pattern of BD interaction shown in Fig. 7 is similar to that of BD interaction for biomass, where the LSD bars do not overlap for either level, indicating that effect of glucose is significant in this interaction. However, for BC, high level of yeast extract causes reduction in DHA accumulation with increasing glucose concentrations. This is in contrast to interaction of BD where high level of MSG promotes DHA accumulation when glucose is at high level.

\subsection{Validation of Significant Factors}

The conclusions drawn from half normal plots above (Fig. 1-5) were verified by performing Analysis of Variance (ANOVA) and the associated diagnostics of residual error as recommended by Anderson and Whitcomb (2007). Table 6 summarizes the softwaregenerated ANOVA results for all three responses. In this analysis, the outstanding effects are incorporated into the "model" and the smaller effects are pooled together to estimate the error called "residual". "Cor total" values are the total sum of squares corrected for the mean. It represents the total system variation using the average response as a baseline (Anderson and Whitcomb, 2007).

On the whole, sea salt was found to have opposite effects on biomass and lipid. Similarly, interaction of glucose and MSG has contrasting effects on biomass and DHA content. Interactions between sea salt-glucose and glucose-yeast extract also exert significant effects on lipid and DHA content respectively. Therefore, all the above mentioned factors, namely sea salt, glucose, yeast extract and MSG are considered as significant factors $\mathrm{P}$
$<0.05$ is significant that have strong influence on DHA accumulation of Schizochytrium sp. SW1. The levels of these vital factors were then restructured to suit the system according to our understanding of cellular physiology of this isolate in relation with the above mentioned effect of factors.

\section{DISCUSSION}

Glucose was chosen as the main carbon source based on the results of reported studies where it is proven that glucose serves as the best carbon source for biomass, lipid and DHA accumulation for most Traustochytrids (Shene et al., 2010). However, in contrast to the general understanding that glucose concentration is directly related to biomass accumulation, this component was found to be statistically insignificant in affecting the final biomass concentration of Schizochytrium sp. SW1. Ganuza and Izquierdo (2007) reported that glucose concentrations beyond $14 \mathrm{~g} \mathrm{~L}^{-1}$ caused only a slight increment in biomass of Schizochytrium G13/2S. This is in agreement with our results where elevation of glucose concentrations up to $60 \mathrm{~g} \mathrm{~L}^{-1}$ does not score a significant effect value (Table 4). However, interaction of glucoseMSG has significant negative effect on the amount of biomass produced. As mentioned above, when both glucose and MSG are at high or low levels, abundance or complete scarcity of both nutrients in combination suppresses cell proliferation to a considerable extent, based on its probability value of 0.02 . This can be related to two different stress phenomenons. Low concentrations 
of both glucose and MSG lead to poor carbon and nitrogen supply which causes starvation. In such condition, cell proliferation is significantly reduced. On the other hand, excess supply of these two components increases the relative osmotic pressure of the culture medium compared to the cellular environment. This hyperosmotic pressure may result in an imbalanced fluid flux which leads to cell shrinkage.

The only major effect that has significant effect on biomass accumulation is sea salt. Increasing concentrations of sea salt up to $35 \mathrm{~g} \mathrm{~L}^{-1}$ (100\% salinity) was found to be statistically very favorable for biomass accumulation based on its positive effect value as shown in Table $4(\mathrm{p}<0.0001)$. Chang et al. (2012) reported similar findings in their study dealing with Aurantiochytrium sp. KRS 101 where biomass yield increased with increasing sea salt concentration which prevents cell rupture. This is directly related to the sampling locations in most cases where habitants of mangrove areas (coastal sea) are moderately halophilic in nature due to constant reduction in salinity due to outflow of rivers. In contrast, habitants of sea water, such as Schizochytrium sp. SW1 are relatively more halophilic due to high salt content of sea water. Reduction in salt concentrations is often related to cell rupture and subsequent biomass loss (Chang et al., 2012). However, high sea salt concentration is not preferred in biotechnological processes due to its corrosive nature caused by the presence of chloride ions (Taha et al., 2013). Therefore, it is desirable to include sea salt in moderate levels where it does not cause significant reduction in biomass. This can be determined by performing a statistical optimization experiment such response surface analysis.

The lipid content (\% lipid/biomass) of Schizochytrium sp. SW1 was found to be affected significantly by the levels of sea salt and the interaction of sea salt-glucose. In contrast to the first response discussed above, lipid content is inversely affected by increasing salt concentrations. This is in agreement with the results obtained by Zhu et al. (2008) where the lipid content of S.limacinum OUC88 was found to decrease with increasing salt concentrations. Sea salt provides vast variety of ions that are essential for normal physiological functions of cells, especially for marine microbes. The production medium used in this study contains sea salt as the sole source of all vital ions. At high sea salt concentrations, essential ions are present in abundance, causing the organism to be less stressed. At such conditions, the tendency of cell proliferation is higher compared to cell enlargement which is mainly due to increased storage nutrients such as lipid. It is known that excess production and accumulation of lipid is mainly a stress-response mechanism where excess carbon that cannot be utilized for cell proliferation (due to other limiting nutrients) will be stored as lipids. Thus high sea salt concentrations promote cell proliferation but not lipid accumulation. Therefore, it has negative impact on the lipid content which is indicated by a negative coefficient in statistical terms. Most studies achieve such stress induction state by limiting the supply of nitrogen sources.

Interaction of sea salt and glucose on the other hand has positive effects on lipid accumulation. However, at high concentrations of glucose, effect of sea salt on lipid is not significant. As the concentration of glucose decreases, impact of sea salt becomes very profound where any slight increase in salt concentration causes steep decrease in the lipid content of the cells (Fig. 3). This can be explained by considering the cells proliferation tendency as mentioned above. When carbon supply is low (low glucose levels) and there is no other limiting factors, all forms of assimilable carbon are channeled to favor cell proliferation and storage lipids (if any) are actively catabolised to meet the carbon demand of the cells. Therefore, it is desirable to maintain glucose concentrations at high level in order to minimize the impact of sea salt concentrations. By doing this, high amounts of both biomass and lipid can be reached without the incorporation of high amounts of salt.

Compared to the two responses discussed above, DHA content of lipid is not significantly affected by any major factor. However, interactions of glucose-yeast extract and glucose-MSG are strong influential factors that affect this response in opposite manner. Interaction of glucose and yeast extract affects DHA content negatively, where high concentration of both components causes DHA content to decrease, meanwhile interaction of glucose-MSG has positive effects. The negative coefficient of glucose-yeast extract (BC) interaction compared to glucose-MSG (BD) most probably implies that $\mathrm{MSG}$ is the preferred nitrogen source compared to yeast extract. This finding is in agreement with that reported by Burja et al. (2006) where supplying the culture of Traustochytrium sp. ONC-T18 with $10 \mathrm{~g} \mathrm{~L}^{-1}$ MSG replacing yeast extract resulted in $43 \%$ increment in DHA content of lipid. However, the biomass production and lipid accumulation were critically low with complete omission of yeast extract. This might be due to lack of vitamins which are otherwise provided by yeast extract. Low amount of yeast extract $\left(2 \mathrm{~g} \mathrm{~L}^{-1}\right)$ and moderate amount of MSG $(8 \mathrm{~g}$ $\mathrm{L}^{-1}$ ) were shown to produce highest biomass, lipid and 
DHA. Similarly, Ren et al. (2013) also suggested that MSG promotes DHA production. However, since high levels of both glucose and MSG are shown to have negative effect on biomass, it is desirable to use moderate levels of MSG in combination with high levels of glucose to increase DHA yield (Burja et al., 2006; Yokochi et al., 1998). This is important to overcome the inhibitory effect of glucose-MSG on the biomass production of this isolate. Nonetheless, at high levels of glucose, concentration of yeast extract should be maintained low to suppress the adverse effect of glucoseyeast extract interactions on DHA content.

Overall, all factors studied, excluding peptone have significant effect on biomass production as well as lipid and DHA accumulation by this isolate. This experiment has revealed that levels of sea salt, yeast extract and MSG should be maintained at moderate levels, whereas glucose should be provided in high concentration to enable optimal biomass production with high proportions of lipid and DHA. This provides a good perception about the significant medium components and the modes of their action in influencing the responses leading to successful medium optimization for enhanced DHA production by Schizochytrium sp. SW1.

\section{CONCLUSION}

Screening of medium components for eficient production of DHA by Schizochytrium sp. SW1 was successfully conducted using two-level factorial design. Four out of the five medium components studied, namely sea salt, glucose, yeast extract and MSG were found to have significant effects on the responses either as a single factor or in combination with another factor. Interpretation of interaction plots enabled determination of effective range of each parameter for improved and accurate optimization processes.

\section{ACKNOWLEDGEMENT}

We thank the Ministry of Higher Education Malaysia for funding this research under the Exploratory Research Grant Scheme-ERGS/1/2012/STG08/UKM/02/15.

\section{REFERENCES}

Anbu, P., S.C.B. Gopinath, A. Hilda, T. Lakshmipriya and G. Annadurai, 2006. Optimization of extracellular keratinase production by poultry farm isolate Scopulariopsis brevicaulis. Biores. Technol., 98: 1298-1303. DOI: 10.1016/j.biortech.2006.05.047
Anderson, M.J. and P.J. Whitcomb, 2007. DOE Simplified: Practical Tools for Effective Experimentation. 2nd Ed. Productivity Press, New York, ISBN-10: 1563273446, pp: 241.

Barclay, W.R., C. Weaver and J. Metz, 2005. Development of a Docosahexaenoic Acid Production Technology Using Schizochytrium: A Historical Perspective. In: Single Cell Oils, Cohen, Z. and C. Ratledge, (Eds.), AOCS, Champaign, Illinois, ISBN-10: 1893997804, pp: 36-52.

Barclay, W.R., K.M. Meager and J.R. Abril, 1994. Heterotrophic production of long chain omega-3 fatty acids utilizing algae and algae-like microorganisms. J. Applied Phycol., 6: 123-129. DOI: $10.1007 / \mathrm{BF} 02186066$

Behrens, P.W. and D.J. Kyle, 1996. Microalgae as a source of fatty acids. J. Food Lipids, 3: 259-272. DOI: $10.1111 / \mathrm{j} .1745-4522.1996 . t b 00073 . \mathrm{x}$

Burja, A.M., H. Radianingtyas, A. Windust and C.J. Barrow, 2006. Isolation and characterization of polyunsaturated fatty acid producing Thraustochytrium species: Screening of strains and optimization of omega-3 production. Applied Microbiol. Biotechnol., 72: 1161-1169. DOI: 10.1007/s00253-006-0419-1

Chang, K.J.L., G.A. Dunstan, G.C.J. Abell, L.A. Clementson and S.I. Blackburn et al., 2012. Biodiscovery of new Australian thraustochytrids for production of biodiesel and long-chain omega-3 oils. Applied Microbiol. Biotechnol., 93: 2215-2231. DOI: $10.1007 / \mathrm{s} 00253-011-3856-4$

Chi, Z., D. Pyle, Z. Wen, C. Frear and S. Chen, 2007. A laboratory study of producing docosahexaenoic acid from biodiesel waste glycerol by microalgal fermentation. Proc. Biochem., 42: 1537-1545. DOI:10.1016/j.procbio.2007.08008

Chi, Z., Y. Liu, C. Frear and S. Chen, 2009. Study of a two-stage growth of DHA-producing marine algae Schizochytrium limacinum SR21 with shifting dissolved oxygen level. Applied Microbiol. Biotechnol., 8: 1141-1148. DOI: 10.1007/s00253008-1740-7

Duta, F.P., F.P. de Franca and L.M.D.A. Lopes, 2006. Optimization of culture conditions for exopolysaccharides production in Rhizobium sp. using the response surface method. Electron. J. Biotechnol., 9: 391-399. DOI: 10.2225/vol9-issue4fulltext-7

Fedorova, D., P.A. Marone, E. Bailey-Hall and A.S. Ryan, 2011. Safety evaluation of algal oil from Schizochytrium sp. Food Chem. Toxicol., 49: 70-77. DOI: $10.1016 /$ j.fct.2010.09.033 
Folch, J., M. Lees and G.H. Sloane-Stanley, 1957. A simple method for isolation of total lipids from animal tissues. J. Biochem., 226: 497-509.

Ganuza, E. and M.S. Izquierdo, 2007. Lipid accumulation in Schizochytrium G13/2S produced in continuous culture. Applied Microbiol. Biotechnol., 76: 985-990. DOI: 10.1007/s00253-007-1019-4

Hauvermale, A., J. Kuner, B. Rosenzweig, D. Guerra and S. Diltz et al., 2006. Fatty acid production in Schizochytrium sp. Involvement of a polyunsaturated fatty acid synthase and a type I fatty acid synthase. Lipids, 41: 739-747. PMID: 17120926

Perez-Garcia, O., M.E. Froylan, L.E.E. Bashan and Y. Bashan, 2011. Heterotrophic culture of microalgae: Metabolism and potential products. Water Res., 45: 11-36. DOI: 10.1016/j.watres.2010.08.037

Ratledge, C., 2003. An opinion on the safety data for the DHA-rich oil from Schizochytrium sp., also known as DHASCO-S. GRAS Notification for DHA Algal Oil Derived from Schizochytrium sp., pp: 5.

Ren, L.J., J. Li, Y.W. Hu, X.J. Ji and H. Huang, 2013. Utilization of cane molasses for docosahexaenoic acid production by Schizochytrium sp. CCTCC M209059. Korean J. Chem. Eng., 30: 787-789. DOI: 10.1007/s11814-013-0020-0

Shene, C., A. Leyton, Y. Esparza, L. Flores and B. Quilodran et al., 2010. Microbial oils and fatty acids: Effect of carbon source on docosahexaenoic acid (C22:6 n-3, DHA) production by thraustochytrid strains. J. Soil Sci. Plant Nutr., 10: 207-216. DOI: 10.4067/S0718-95162010000100002

Taha, A.I.B.H.M., T. Kimoto, T. Kanada and H. Okuyama, 2013. Growth optimization of thraustochytrid strain $12 \mathrm{~B}$ for the commercial production of docosahexaenoic acid. Food Sci. Biotechnol., 22: 53-58. DOI: 10.1007/s10068-0130048-2
Tumpang, N.H.M., M.M. Salleh and S.A. Aziz, 2011. Screening of factors influencing exopolymer production by Bacillus licheniformis strain T221a using 2-level factorial design. Progress Mol. Environ. Bioeng. Anal. Model. Technol. Applicat. DOI: $10.5772 / 19574$

Vazhappilly, R. and F. Chen, 1998. Eicosapentaenoic acid and docosahexaenoic acid production potential of microalgae and their heterotrophic growth. J. Am. Oil Chemists' Soc., 75: 393-397. DOI: 10.1007/s11746-998-0057-0

Wu, S.T., S.T. Yu and L.P. Lin, 2005. Effects of culture conditions on docosahexaenoic acid production by Schizochytrium sp. S31. Proc. Biochem., 40: 31033108. DOI: 10.1016/j.procbio.2005.03.007

Yokochi, T., D. Honda, T. Higashihara and T. Nakahara, 1998. Optimization of docosahexaenoic acid production by Schizochytrium limacinum SR21. Applied Microbiol. Biotechnol., 49: 72-76. DOI: $10.1007 / \mathrm{s} 002530051139$

Zeng, Y., X.J. Ji, M. Lian, L.J. Ren and L.J. Jin et al., 2011. Development of a temperature shift strategy for efficient docosahexaenoic acid production by a marine fungoid protist, Schizochytrium sp. HX-308. Applied Biochem. Biotechnol., 164: 249-255. DOI: 10.1007/s12010-010-9131-9

Zhu, L., X. Zhang, X. Ren and Q. Zhu, 2008. Effects of culture conditions on growth and docosahexaenoic acid production from Schizochytrium limacinum. J. Ocean Univ. China, 7: 83-88. DOI: 10.1007/s11802008-0083-4 